Vol.15, No. 57, October, 2020, 1178-1198

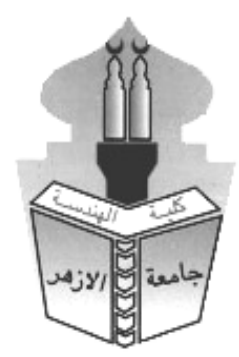

\title{
DEVELOPMENT OF MULTI-MODULE ARTIFICIAL MUSCLE USING HYBRID ACTUATOR
}

\author{
Hend Mahmoud Hamdi ${ }^{*}$, Sabreen Abdullah Abdelwahab ${ }^{2}$, and Mohammed Ibrahim \\ Awad $^{3}$ \\ ${ }^{1}$ Design and Production Engineering Dept., Faculty of Eng,, Ain Shams University, Cairo, Egypt. \\ ${ }^{2}$ Production Technology Dep, Faculty of Industrial Education, Helwan University, Cairo, Egypt. \\ ${ }^{3}$ Mechatronics Engineering Dept., Faculty of Engineering, Ain Shams University, Cairo, Egypt. \\ *Corresponding Author E-mail: hend.hamdi@eng.asu.edu.eg
}

\begin{abstract}
:
Biological muscle is considered an inspiring actuator for the researchers in the field of biorobots. The main building unit of a muscle, sarcomere, and the study of its energy cycle is analyzed to mimic the micro-level muscle components' behavior to improve actuator performance and efficiency. A newly developed hybrid material actuator is designed using a combination of Ionic Polymeric Metallic Composites and hydrogel to behave like the sarcomere with different deflection types. The proposed module is arranged in two different configurations and performance is investigated for working at once or working in sequence. A physical model is proposed, and a mathematical model of the actuator is derived and formulated using MATLAB Simulink. The proposed arrangements show an improvement in force and displacement magnification of three times relative to the single module. Also, working in a sequence shows promising results to work effectively to simulate muscle performance for motion profiles.
\end{abstract}

KEYWORDS: Artificial Muscle, Ionic Polymeric Metallic Composites, Hydrogel, Hybrid Actuator, and Multi-Module Actuator.

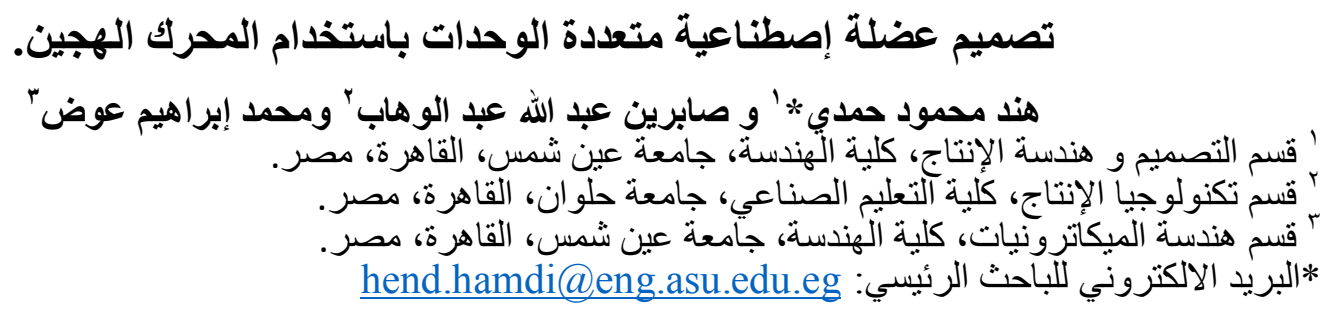

\footnotetext{
(الملخص:

تعتبر العضلات البيولوجية محركًا ملهمًا للباحثين في مجال الروبونات الحيوية. يتم تحليل وحدة البناء الرئيسية للعضلة،

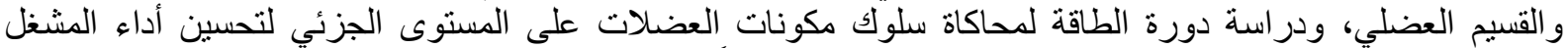

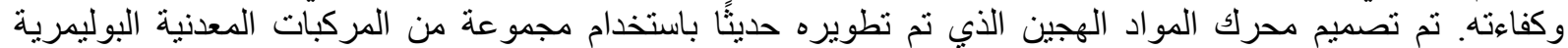

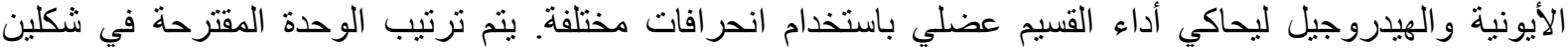
مختلفين ويتم فحص الأداء للعمل دفعة واحدة أو للعمل في تسلسل. تم اقتر اح نموذج الفيزيائي ، وتم اشتقاق نموذج الَرياضي
} 


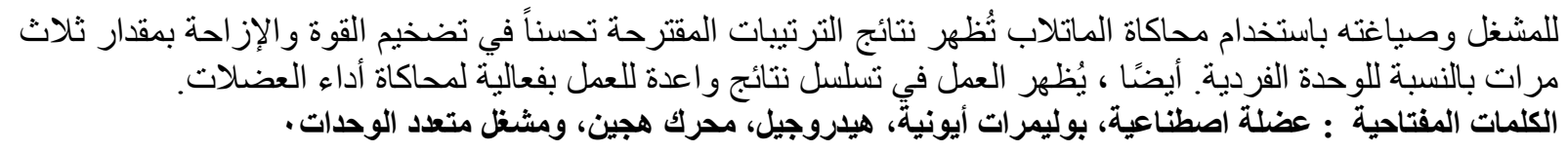

\section{INTRODUCTION}

Bioinspired artificial muscles have been recently escalated to cover numerous fields, as the continuous improvement of automated systems requires efficient energy utilization [1], as the conventional actuators have shortcoming in power to weight proportion [2], [3]. The natural muscle is considered an energetic source of inspiration for its multi-tasking actuation, flexible modular design, and competent energy utilization [2]. Many models were developed to characterize the natural muscle behavior such as Hill and Huxley models, which are extensively used. It was found that analyzing the muscle behavior on the micro-level and modeling the sarcomere behavior improves the results smoothness, so in this study we will focus on the sarcomere main chemical contraction cycle, the four-steps Adenosine Tri Phosphate (ATP) cycle which is the reason of the contraction mechanism.

The development of Electro Active Polymers (EAP) supports to improve the bioinspired actuators designs. They show a variety of mechanical response as elongation, bending, buckling, and volumetric change under the effect of different types of physical stimulators [4]. EAP is divided into two main categories, electronic and ionic. Ionic Polymeric Metal Composites (IPMC) is a class of ionic polymers that transforms the input electrical voltage into bending displacement [5]. This ability was used to model the movement of muscle proteins in the sarcomere [6]. Throughout this study, this concept is expanded by incorporating a chemically induced hydrogel terminal that can shrink, stretch, and transfer into different binding sites. The proposed hybrid material actuator simulates the sarcomere's four-state ATP actuation process.

\subsection{Biological Motivation}

Natural skeletal muscle governs the human body motion. It is responsible for preserving bones and joints stability and avoids extreme movements that may cause injury or distortion[7]. The detailed structure of the human skeletal muscle is shown in Fig. 1.

Sarcomere contraction is achieved by cross-bridge creation between the main active proteins in the sarcomere unit, myosin heads, and actin filaments. The initiation of this cycle depends on the nervous system which pumps calcium ions $\mathrm{Ca}++$ to start motion excitation. Sarcomere shortening starts when actin is moved by myosin and then binding sites appear for engagement during the power stroke. ATP molecules are necessary to continue the contraction process. As the calcium ions, $\mathrm{Ca}++$ are removed, contraction stops, and the binding sites are sheltered again by tropomyosin [8].

The analysis of the muscle contraction process energy balance requires to go through the sarcomere's micro muscle stage and a short explanation of the actin-myosin binding process. The trajectories of force and muscle length over time have been proven to be smoother and more relevant in the case of using Huxley's muscle model rather than Hill's model, which proves that microscopic level modeling is more suitable [9].

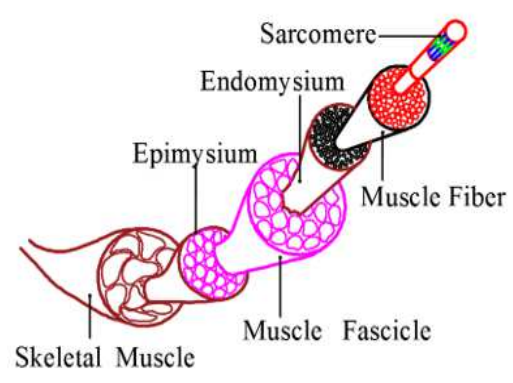

(a)

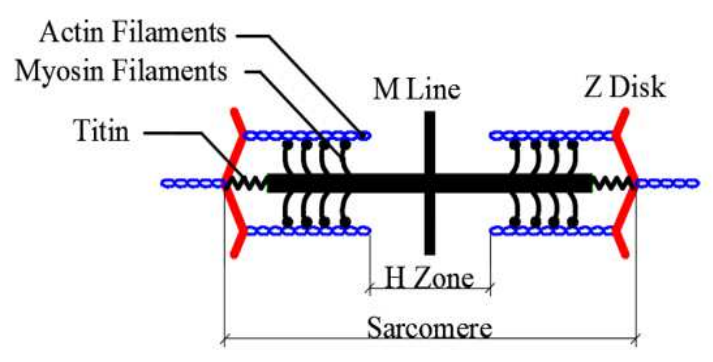

(b)

Fig. 1. (a) Natural Muscle Details (b) A single sarcomere unit.

The muscle contraction cycle is directly linked to the ATP hydrolysis mechanism, which is the major source of energy consumed. In the lack of calcium, no action will be taken on either 
sarcomere motor protein, actin, or myosin. As $\mathrm{Ca}++$ binds the actin sites, the cycle starts. The ATP loop was demonstrated by Lymn and Taylor as follows [10],

$$
\mathrm{ATP} \rightleftharpoons \mathrm{ADP}+\mathrm{Pi}+\text { energy }
$$

where ADP (Adenosine Di Phosphate) is responsible for the actin and cross-bridge forming of the myosin head connection. The resulting energy allows the actin to move forward. The ATP hydrolysis induces the myosin head to initiate the power-stroke, which allows the actin to slide between the actin filaments and the myosin head [11].

The one- direction myosin head movement from a binding site along the actin to another is divided into four main steps: Disengagement of actin and myosin and a new ATP molecule is delivered, preparing for the new step forward, which called recovery stroke. Engagement of actin and myosin at the actin binding sites. The myosin head moves forward pulling the actin filament using the released energy coming from ATP hydrolysis [12]. The coupling of the mechanical and chemical cycles of the motor proteins is illustrated in Fig. 2, showing the power and return stroke synchronized with the ATP hydrolysis [13]. Most of the preceding studies concluded that four mechanical conditions arising from the ATP hydrolysis reaction were present.

Since the natural sarcomere working cycle has four steps, the myosin can work in a sequence to ensure that the actin is subjected to tension along the working time needed to accomplish the contraction. Besides, the number of myosin heads involved in the contraction depends on the effort needed by the muscle. The invention for modular actuator design presented in this work helps to study the abovementioned phenomena to mimic the natural muscle behavior. The design flexibility enables studying different possible configurations.

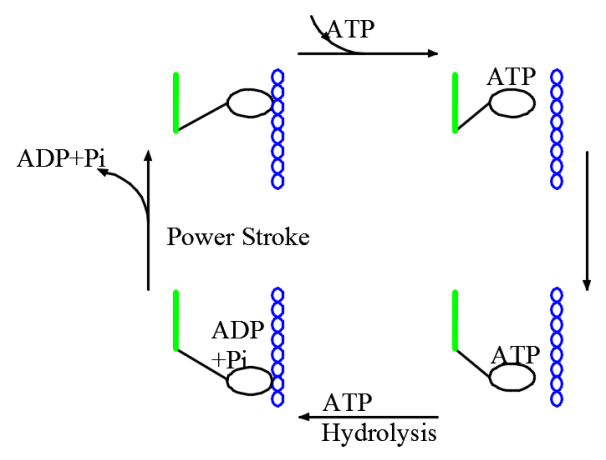

Fig. 2. The Four Steps Chemical Cycle of ATP and The Relating Contraction Cycle.

\subsection{Artificial Muscles Modular Designs:}

Modular artificial muscles are commonly used in the field of the biorobots. Many designs have been developed to efficiently resemble the natural muscular behavior and focused on the modular nature of the skeletal muscle. Huxley's model was simulated in[14] using a hardware model to act like a sarcomere. This work was extended by building a series of actuators in order to study the steady-state and transient behavior of a sarcomere on a muscle fiber [15]. Also, it was used in autonomous robots [16].

Ebrahimi et al. [17] proposed a series of elastic soft actuator array to enhance safe interaction with users, as they are able to reduce large force due to sudden loading. Schultz et al.[18] showed the benefits of modularity of actuator design in changeable actuators recruiting strategies. Their work was extended in [19] to modify actuator modular actuator impedance produced by exploiting the redundancy in control inputs. Jing et al. [20] proposed a combined series-parallel linear actuator is based on electromagnetic technology as a result of analysis of biological muscle structure and function, they stated that the actuator has good prospects for actual engineering application and it is applied on the multi-legged robot in [21] and the output force is estimated.

While several forms of biomimetic actuators have been designed to behave like the natural muscle, electroactive polymers (EAPs) have also been found to be the closest type to real behavior, because they have a similar range of actuating energy, the same deflection structure, and the most appropriate mechanical work cycle needed to have the necessary chemical cycle 
steps [2]. The two main groups are electronic EAP and ionic EAP, the second is chosen due to lower required activation voltage and higher subsequent displacement. A hybrid material actuator is proposed in [22] to behave like a myosin unit during the power stroke and return, since the behavior of each material results in different deflection under activation.

It was noted in the previous researches there is a problem with output force relative to actuator weight. Also, the utilization of hybrid material is still limited although it provides different types of responses to the actuator with different motion possibilities.

In the current research, a changeable recruitment multi-module actuator is presented to manage load changes efficiently. The proposed actuator [23] is placed in two new configurations combining a number of actuating modules in predefined arrangements to enhance the resultant performance given by one module. As shown from previous work [23], each module is considered hybrid material actuation element which is the electrically activated IPMC acting as a myosin lever arm, and the chemically actuated hydrogel terminal acting as a myosin head.

\subsection{Hydrogels and Swelling Reaction:}

Hydrogels are elastic materials that have the ability to change or increase volume if it is actuated by a stimulator such as chemical, electrical, magnetic, or thermal. This volumetric change is called swelling, which enables the hydrogel to be utilized in biomimetic actuators design. Chemically stimulated gels by means of oscillatory chemical reaction or varying $\mathrm{pH}$ solution value give more powerful results rather than when it is electrically stimulated [24]. To perform these chemical reactions, the gel is immersed into a solvent or varying chemical environment, which results in cation and anion exchange between gel and surrounding solution [25].

The oscillatory Belousov-Zhabotinsky (BZ) reaction explored by Yoshida et al. in [26] is one of the chemical stimulation techniques used for autonomously generating cycles of oxidation and reduction needed for hydrogel swelling. This reaction is preferred than the $\mathrm{pH}$ stimulation since it doesn't need any external control for the reaction equipment [27]. This cyclic stimulation was applied to develop a chemo-mechanical actuator. The generated chemical wave and the resultant osmosis pressure from the wave propagation inside the gel were utilized to actuate a chemical robot that can translate an object[28].

Fig. 3 shows the effect of the generated chemical wave due to the reaction on the polymer starting from the monomer oscillation, reaching the bulk gel wave propagation. The resulting gel behavior depends on the gel sample size and its relationship with the chemical wavelength [29].

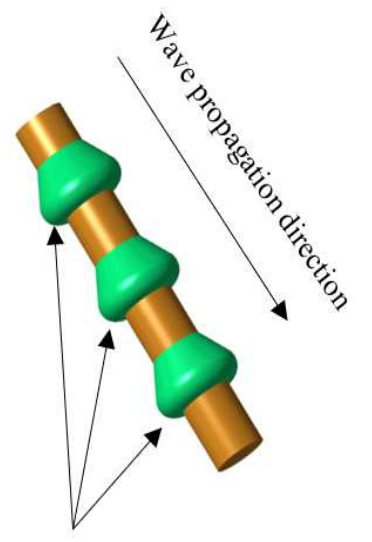

Swelling Regions

Fig. 3. The chemical wave generated by the $\mathrm{BZ}$ reaction and the resulting swelling shape in the hydrogel.

The abovementioned behavior is utilized in our work to generate an autonomous voltage signal through a hydrogel element that operates the IPMC beam bending, which in turn moves the actuator slider. The beating gel head turns on the operating switch while swelling and the 
switch is turned off while deswelling. Also, the head engages with the slider enabling the IPMC beam to push the slider forward.

\subsection{Ionic Polymeric Metallic Composites (IPMC's):}

IPMC's are widely used in most of the soft robots' applications since they can be activated by electrical energy into mechanical energy in a form of bending used in actuation. The basic advantage of using IPMC that it produces a relatively large deflection when a low voltage is applied[30]. It is consisting of a thin Nafion membrane, covered with two platinum electrodes on each side [31].

The IPMC shape is a polymeric cantilever membrane contains fixed anions that exist on the polymer chain, free cations, and water molecules. By applying a voltage as shown in Fig. 4 the hydrated cations are transferred to the negative electrode side. Consequently, the osmosis pressure raises at this side results in overall membrane bending displacement [32] [33].

This previously illustrated energy transformation from electrical voltage to mechanical bending of the IPMC beam was modeled to determine its tip displacement and resultant tip force needed for actuator movement. The previously developed model used in [23] is applied in this work to study the developed actuator movement characteristics. The applied voltage on the IPMC strip results from the hydrogel head expansion which gives a sequence of voltage pulses with variable duration controlled by varying the initial concentration of the acids. The proposed design details will be discussed in the following section.

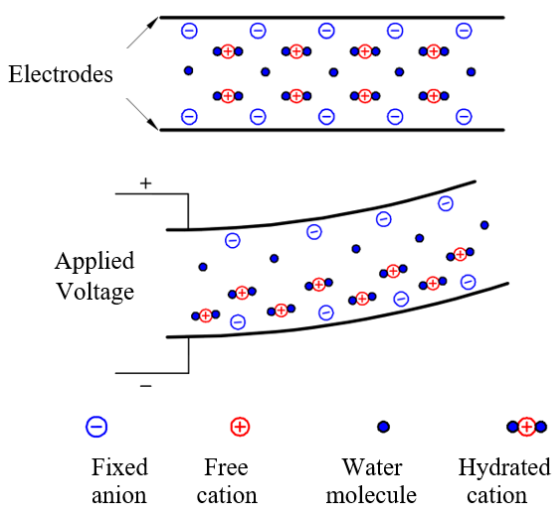

Fig. 4. The effect of electrical activation of the IPMC membrane.

\section{PROPOSED MODULAR ACTUATOR:}

The developed actuator is a modular sarcomere-like structure that simulates the four states cycles of muscle contraction described above. It is an extension of the work proposed in [6]. The concept is expanded to satisfy the planned autonomous cyclic motion specifications by incorporating the hydrogel terminal as the actuator head. The gel terminal is influenced by the cyclic oxidation-reduction caused by the Belousov - Zhabotinsky (BZ) reaction and the wave spreads around its external face. The working sequence of the actuator is shown in Fig. 5. A single module is explained below, then modules are combined in different configurations to enhance the actuator performance measures.

The key actuating feature of the proposed actuator is the IPMC strip terminated with the cyclic swelling of the hydrogel head, which regulates the on-off switch during the swellingdeswelling process cycle. The switch is located inside the slider so that it is in contact with the gel wave at the middle of the cycle, as illustrated in Figure 5, enabling it to move forward and return to the bending strip as a result of the electrical activation of the electrodes located at the bottom of the support. The gel heads are loosely placed in slider slots. 

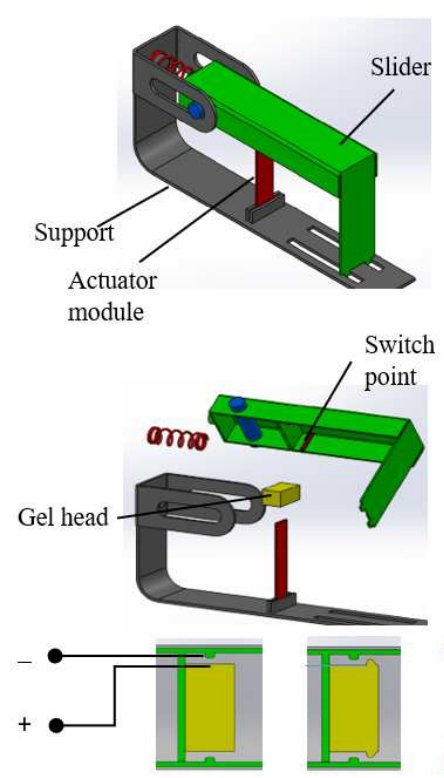
point
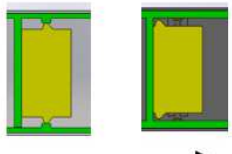

Sequence of wave propagation along the gel head side and relative position to contact point during cycle (top view).

Fig. 5. The proposed actuator mechanical model [23].

At the time of interaction between the gel wave and the slider attachment point, an electrical voltage signal is provided to the electrode connected to the IPMC strip terminal, which starts to bend. As the wave moves away from the switching point, the voltage signal is lost, causing the IPMC flapper strip to return to the idle state. Since the gel head is mounted on the slider, it performs a forward movement during the bending process and then remains at the new position. The gel head is loose during the return stroke and the IPMC strip returns to its original position. This loop can be repeated, causing the slider to push forward rather than onoff and to fluctuate between two stages.

The first proposal was presented in [23], and the performance of one module is investigated. There are many possible configurations and combinations can be given by this design. Adding actuating modules embedded to actuate the same slider or separate sliders. Also, due to the nature of the activation using the chemical reaction, the starting time and duration for each module can be controlled separately. So, in this work two configurations will be investigated and analyzed in order to gain recruitment flexibility to accomplish wide performance. The first arrangement, combined, is done by adding more actuating element to the unit to enlarge output, and the second arrangement is done by adding more sliding modules to the system. They are modeled as shown in Fig. 6.

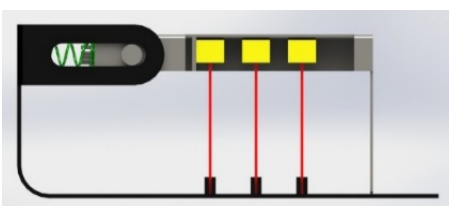

(a)

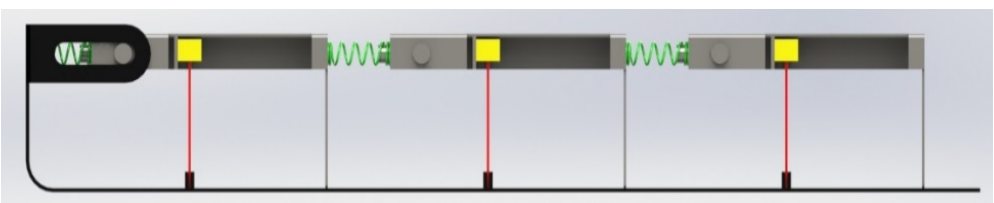

(b)

Fig. 6. New module configurations (a) Combined arrangement (b) Separate arrangement.

\section{MATHEMATICAL MODELING}

In this section, a governing equation of motion is estimated for each of the previously mentioned modules arrangements. To estimate the required equation first we need to explore the mathematical modeling needed to represent the module. This is done on four steps starting from the B-Z reaction needed to generate the main hydrogel actuating wave, then the performance electrical part of IPMC due to electrical activation is estimated, this results in 
mechanical deflation of the IPMC beam, and then it moves the actuator slider at last as shown in Fig. 7.

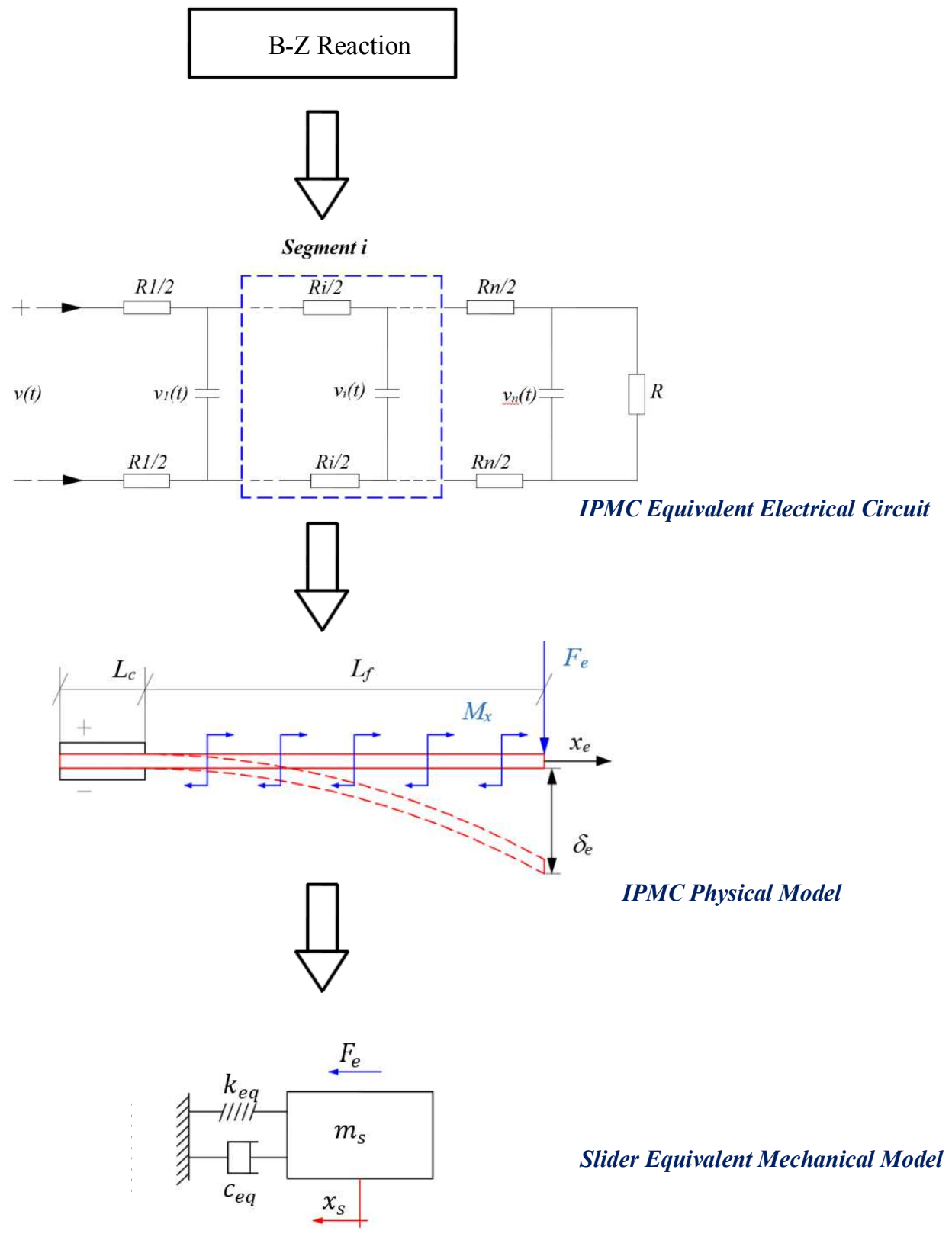

Fig. 7. One Module Actuator Physical Model.

\subsection{Modeling of Hydrogel Head Swelling Wave}

The mathematical model of the BZ chemical reaction is presented in equations (2) to (5), $\frac{a u}{a t}=F(u, v, \phi)$

$\frac{d v}{d t}=G(u, v, \phi)$

$F(u, v, \phi)=(1-\phi)^{2} u-u^{2}-(1-\phi) f v\left[u-q(1-\phi)^{2} / u+q(1-\phi)^{2}\right.$

$G(u, v, \phi)=\varepsilon\left[(1-\phi)^{2} u-(1-\phi) v\right]$ 
where $\mathrm{u}$ is the stimulus component $(\mathrm{HBrO} 3), \mathrm{v}$ is the oxidized ion of the metal in polymer chain $(\mathrm{Ru} 3+),, \mathrm{F}, \mathrm{G}$ are the functions of reactants rate, $\mathrm{f}, \mathrm{q}$, and $\varepsilon$ are dimensionless reaction parameters, and $\psi$ is the resultant volumetric fraction which is responsible for swelling and deswelling [34]. The resulting increase in gel volumetric fraction causes the head width to expand and then increase in length. This gel swelling traveling wave can be modeled as a sine wave mentioned in equation (6) to describe the change in length with time and position [26].

$Z_{o x}(x, t)=\cos ^{2} \pi(x-t)$

This function is dependent on wave periodic time and wavelength, it can be described by the following dimensionless mathematical presentation:

$1=2 \int_{0}^{1} z_{o x}(x, t) d x=2 \int_{0}^{1} z_{o x}(x, t) d t$

Assuming there is no residual gel length at the edge, the resulting gel mechanical wave can be modelled as follows;

$L(t)=2 \int_{0}^{l_{o}} Z_{o x}(x, t) d x$

where $l_{0}$ is the average value of gel length can be calculated as follows;

$L_{o}=\int_{0}^{1} L(t) d t$

The resultant wave propagation surface along the gel head edge and the resultant start and end times for the input voltage signal will be discussed in section 4.

according to physical considerations based on IPMC geometry as follows;

$R_{t}-2 \rho_{l} b / L w, C_{t}-\varepsilon_{t} L w / 2 b$

where $\rho_{1}$ is the electric resistivity of segment $i$ material, $\varepsilon_{i}$ is electric permittivity of element $i$, $L, w$ and $\mathrm{b}$ are the segment length, width, and thickness respectively.

To determine the discharge $Q(t)$, produced by the input voltage $v(t)$, the generalized equation of the electrical model is formed according to Kirchhoff's current law at the segment $i$ loop;

$C_{i} v_{i}=\frac{v_{i}-v_{i+1}}{\pi_{i 11}}-\frac{v_{i+1}-v_{i+2}}{n_{i \mid 2}}$

When this model has low-frequency working conditions, it is convenient to reduce the model complexity and consider the number of elements $n=2$ [38]. Then, the relation between the input voltage $v(t)$ and the absorbed current $i(t)$ can be modeled in the Laplace domain as follows;

$\frac{I(s)}{V(s)}=\frac{a s^{2}+b s+1}{c s^{2}+d s+s}$

Then, the discharge can be estimated from the transfer function;

$\frac{Q(s)}{V[s]}=\frac{a s+b}{c s^{2}+d s+c}$

where,

$a-R R_{2} C_{1} C_{2}$

$b-R C_{1}+R_{2} C_{1}+R C_{2}$

c $-R R_{1} R_{2} C_{1} C_{2}$ 
$d-R_{1} R_{2} C_{1}+R R_{1} C_{1}+R R_{1} C_{2}+R R_{2} C_{2}$

$-R+R_{1}+R_{2}$

This discharge causes a stress distribution across the cross-section of the IPMC beam, which in turn creates a moment. The IPMC beam deflects due to the bending moment applied and it moves forward with a displacement $\delta$ [39]. The beam bending displacement $\delta$ with no load results from the rearrangement of water molecule inside the beam- is determined by equation $(15)$

$\delta(t)=\hat{k}_{v} W(t)=4 \hat{k}_{v} Q_{i}(t)$

where $k_{v}$ is deformation coefficient, $W(t)$ is water molecules concentrations [40].

Then we take the Laplace transformation for equation (15) and get,

$\delta(s)=k_{v} W(s)=4 k_{v} Q_{i}(s)$

Substitute from (13) into (16) we get,

$\delta(s)=4 k_{v} \frac{a s+b}{c s^{2}+d s+e} V_{l}(s)$

To estimate the IPMC tip force, the relationship between the tip deflection $\delta_{x}$ and distributed moment generated along the strip $M_{x}$ is given by;

$\delta_{x}(t)=M_{x}(t) \cdot x\left(l_{f}-\frac{x}{2}\right) / E I$

So, the total induced bending moment $M_{1}(t)$ is given by;

$M_{l}(t)=\int M_{x}(t) d x=F_{e}(t) \cdot L_{f}$

Then the tip force $F_{e}(t)$ can be estimated as follows;

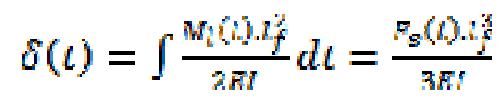

$\delta(s)=\frac{M(s) L_{f}^{2}}{2 L I s}=\frac{H_{\mathrm{e}}\left(s^{2}\right) L^{2} f}{3 t I}$

Then, $F_{\varepsilon}$ can be calculated as follows;

$F_{Q}(s)-\frac{3 E I}{L_{f}^{3}} \delta(s)$

\subsection{Actuator Configurations Modeling}

The lumped models for the proposed configuration are illustrated in Fig. 8. As the slider connected to the IPMC beam end, considering the tip force as an input to the slide, then the generalized form of the set of equations of motion for each configuration can be written as follows,

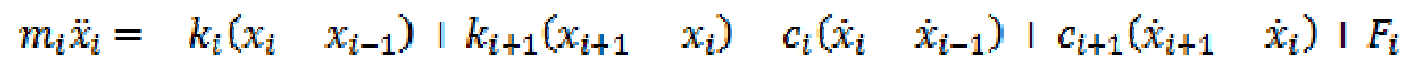


$i=1,2,3$

where $[m],[C],[k]$ are the mass, damping, and stiffness matrices respectively, and are given by,

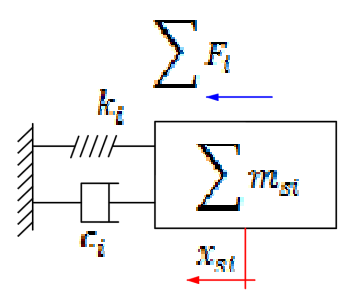

(a)

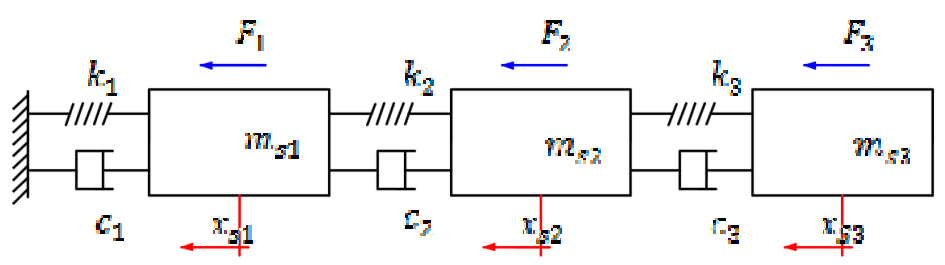

(b)

Fig. 8. Model of the proposed configurations (a) Combined arrangement (b) Separate arrangement.

$$
\begin{aligned}
{[m] } & =\left[\begin{array}{cccccc}
m_{1} & 0 & 0 & & 0 & 0 \\
0 & m_{2} & 0 & \cdots & 0 & 0 \\
0 & 0 & m_{3} & & 0 & 0 \\
& \vdots & & \ddots & & \vdots \\
0 & 0 & 0 & \cdots & 0 & m_{n}
\end{array}\right] \\
{[c] } & =\left[\begin{array}{ccccccc}
\left(c_{1}+c_{2}\right) & -c_{2} & 0 & & 0 & 0 \\
0 & \left(c_{2}+c_{3}\right) & -c_{3} & \ldots & 0 & 0 \\
0 & -c_{3} & \left(c_{3}+c_{4}\right) & & 0 & 0 \\
0 & \vdots & 0 & 0 & \cdots & -c_{n} & \left(c_{n}+c_{n+1}\right)
\end{array}\right] \\
{[k] } & =\left[\begin{array}{ccccccc}
\left(k_{1}+k_{2}\right) & -k_{2} & 0 & & & 0 & 0 \\
0 & \left(k_{2}+k_{3}\right) & -k_{3} & \ldots & 0 & 0 \\
0 & -k_{3} & \left(k_{3}+k_{4}\right) & & 0 & 0 \\
0 & \vdots & 0 & 0 & \cdots & -k_{n} & \left(k_{n}+k_{n+1}\right)
\end{array}\right]
\end{aligned}
$$

Equations (23) to (26) are applied on the preceding physical model for each arrangement to solve for motion criteria using MATLAB Simulink.

\section{SIMULATION AND RESULTS}

In order to analyze the proposed actuator performance, wave propagation behavior is analyzed to calculate the voltage activation and deactivation time. The times of start and end of voltage determine the duration of the IPMC input signal. The signals given to each actuator are triggered with time delays $\tau_{1}$ and $\tau_{2}$ which are first set to zero, which means the performance is tested all at once operation and successive triggering operation. In this work, $\tau_{1}$ and $\tau_{2}$ are set equal to each experiment operating conditions.

The mathematical model of the resultant input sequence of signals, the electromechanical system, and the governing equation of motion for each arrangement using MATLAB Simulink.

To determine the wave propagation profile with time and edge length, equation (6) is solved and results in the dimensionless presentation shown in Fig. 9. The gel head edge at which the 
wave is created has a width of $5 \mathrm{~mm}$. The slider switch point position is adjusted at the middle of the gel head and its width is set so that the duration time is $0.25 \%$ of each cycle.

Fig. 10 shows the chemical wave profile as it reaches and leaves the contact point. The cycle time of the wave depends on the malonic acid concentration in the reaction beginning, the cycle time $T$ in this work is set to $60 \mathrm{sec}$. It results in $t_{1}=15 \mathrm{sec}$. and $t_{2}=15 \mathrm{sec}$. the resultant voltage input pulses for one operating cycle is illustrated and then it is simulated with a signal builder block inside the Simulink model. The reaction start is set for each actuator module all at once or separated by a delay time between every two modules. Here the delay times $\tau_{1}$ and $\tau_{2}$ are first set to zero, equal to, less than, and greater than signal time $t_{2}$.

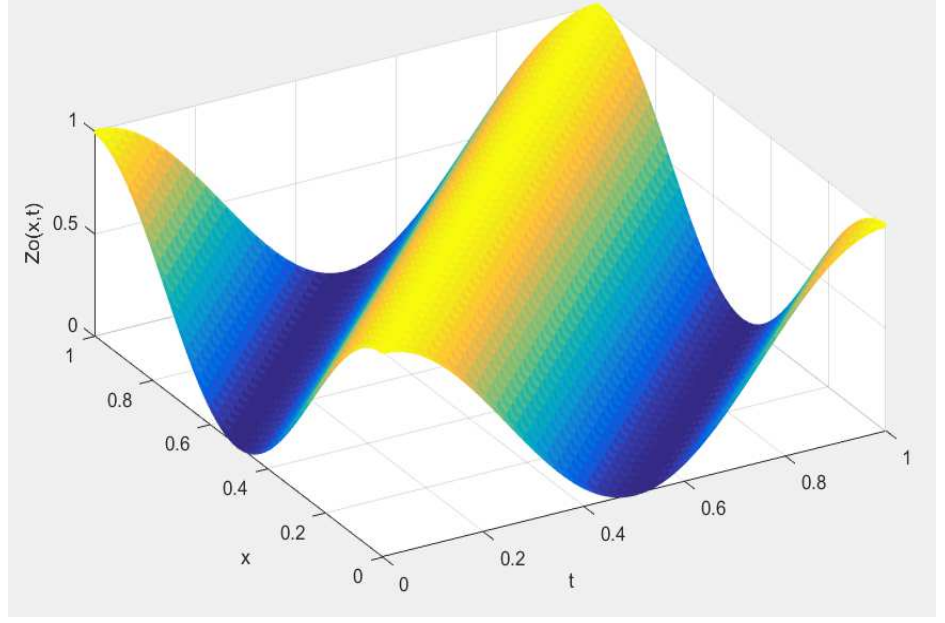

Fig 9. Chemical wave propagation along the gel head edge along distance and time.

Then, the previously estimated IPMC mathematical model is built on MATLAB Simulink with the input voltage signal results from the previous stage. A pulse of a voltage of 1 volt is given to the system simulation as an input signal starts at the previously determine times. The model is estimated for an IPMC strip deflection which addresses the IPMC deflection due to input voltage at no load at the tip. The transfer function parameters $(\mathrm{a}, \mathrm{b}, \mathrm{c}, \mathrm{d}, \mathrm{e})$ are estimated using system identification toolbox with one zero and two poles to match the estimated model configuration [23]. To extract the new configurations of actuator arrangements performance, the mathematical model is extended to find the resultant sliders' displacement, velocity, and force relative to the input voltage signal. The model parameters are estimated based on the mechanical and electrical properties of the moving parts given in Table 1.

Fig. 11 to 18 shows the resultant performance criteria for the proposed actuators arrangements when they are working all at once, which means no delay between voltage signals sent to the three modules, then $\tau_{1}, \tau_{2}=0$ which are the delay times between signals as shown in Fig. 10. Each arrangement is tested under different sequences of time delay combinations to explore the performance under wide varieties of the desired performance range. Then the values of $\tau_{1}, \tau_{2}$ are set to $5,15,20$ seconds which are less, equal, and larger than activation time $t_{2}$ respectively. 
DEVELOPMENT OF MULTI-MODULE ARTIFICIAL MUSCLE USING HYBRID ACTUATOR

Table 1. Model Mechanical parameters.

\begin{tabular}{ll}
\hline Parameter & Value \\
\hline Nafion Density $\left(\mathrm{kg} / \mathrm{m}^{3}\right)$ & 2100 \\
\hline Electrode Density $\left(\mathrm{kg} / \mathrm{m}^{3}\right)$ & 19300 \\
\hline$E(\mathrm{~Pa})$ & $8^{*} 10^{7}$ \\
\hline$B(\mathrm{~m})$ & 0.000032 \\
\hline$L^{*} w(\mathrm{~m} 2)$ & $0.03 * 0.005$ \\
\hline$m_{s}(\mathrm{~kg})$ & 0.002 \\
\hline$k_{v}$ & 0.075 \\
\hline$k_{e q}(\mathrm{~N} / \mathrm{m})$ & 0.01 \\
\hline$D_{e q}(\mathrm{N.s} / \mathrm{m})$ & 0.01 \\
\hline
\end{tabular}

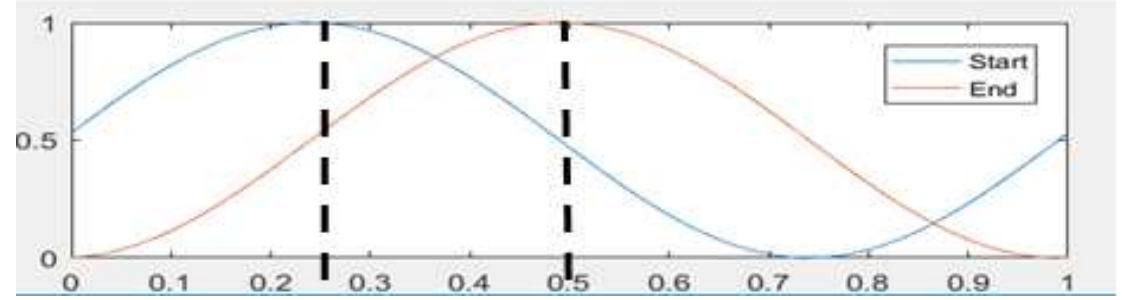

(a)

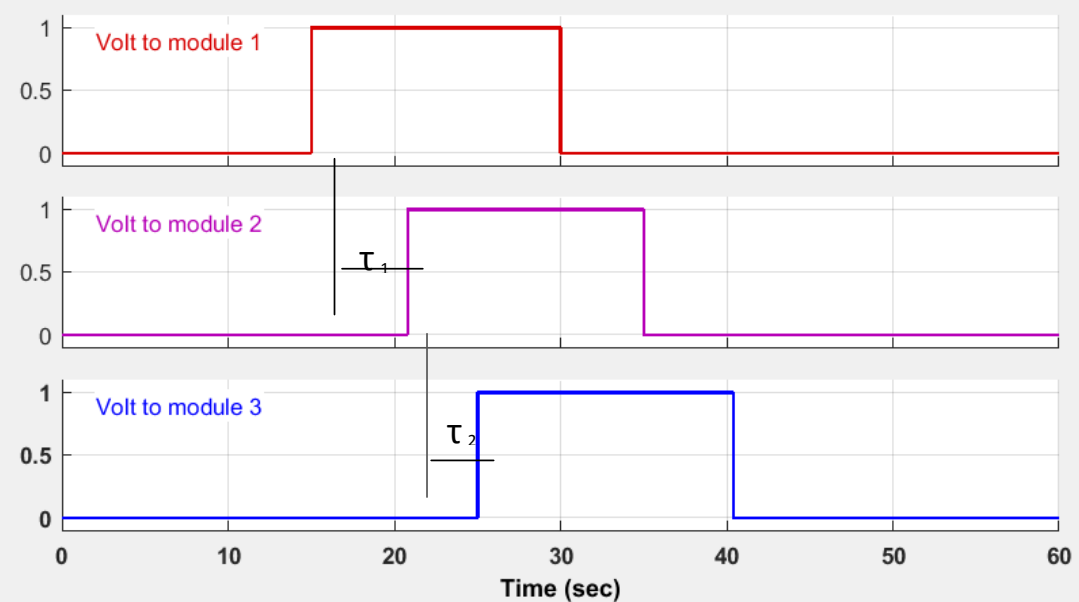

(b)

Fig. 10. (a) Wave profiles at signal start and end (b) The resultant voltage signal for one cycle for every module 
DEVELOPMENT OF MULTI-MODULE ARTIFICIAL MUSCLE USING HYBRID ACTUATOR
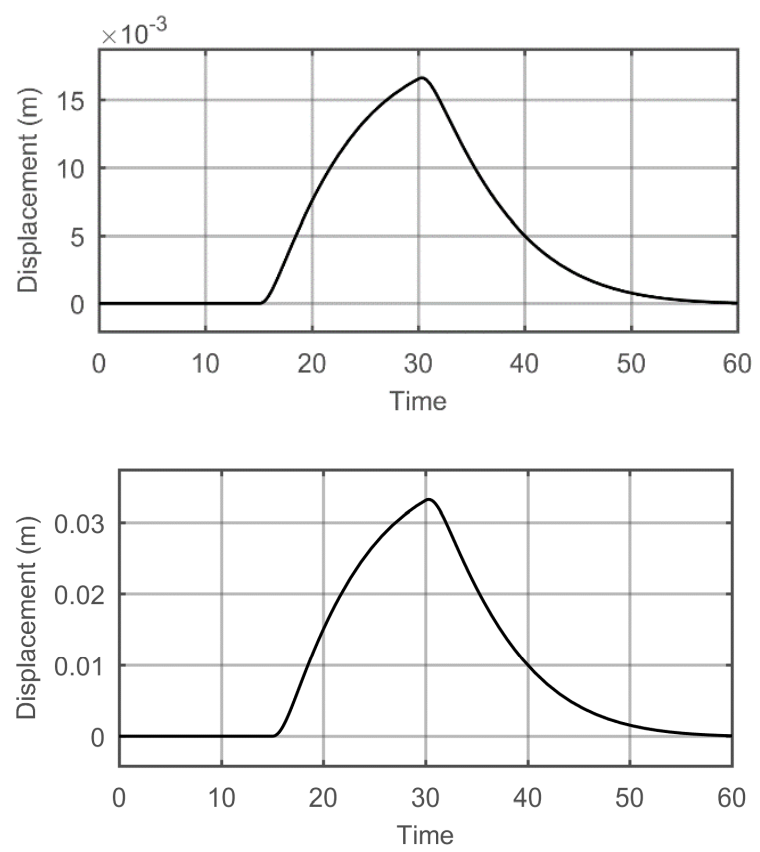

(a)

(a)
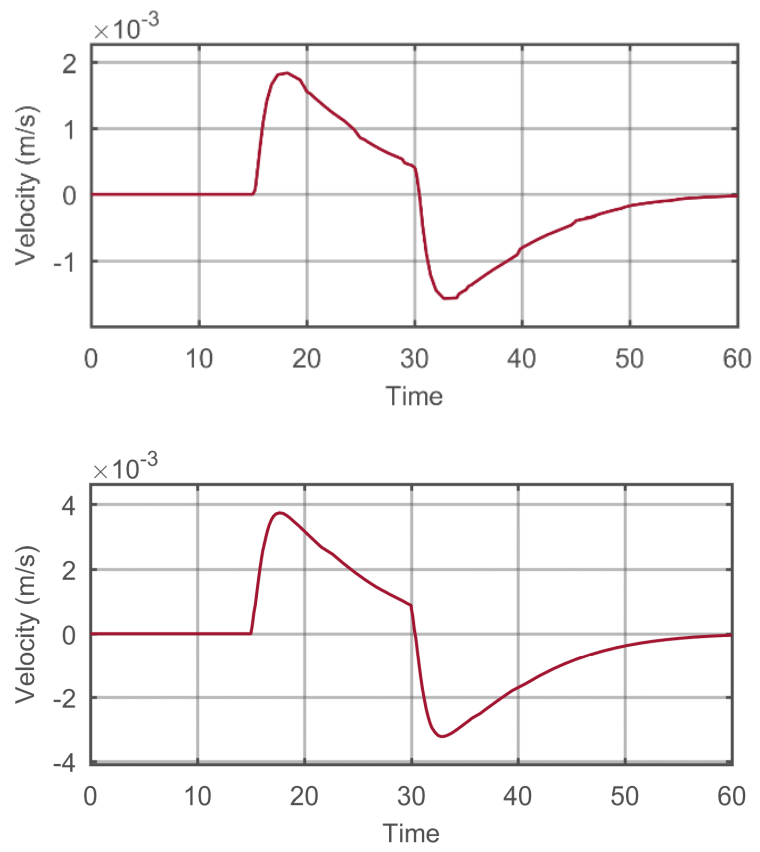

(b)

(b) 

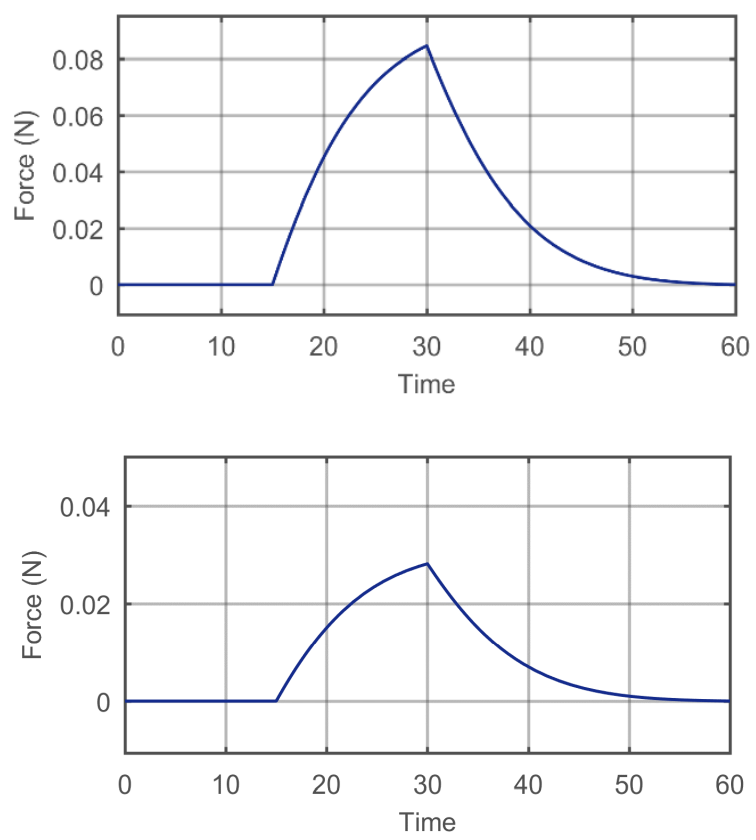

(c)

Fig. 11. Modules combined arrangement results at $\tau_{1}, \tau_{2}-0$ ser. (a) Displacement (b)

Velocity (c) Force.
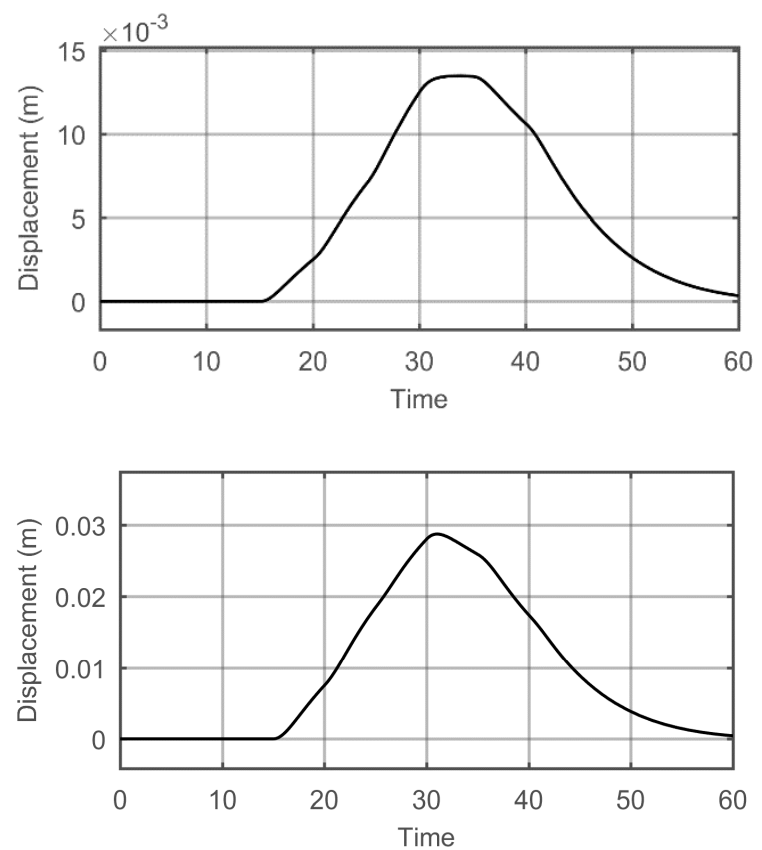

(a) (c)

Fig. 12. Modules separate arrangement results at $\tau_{1}, \tau_{2}=0$ sec. (a) Displacement (b) Velocity (c) Force. (a) 

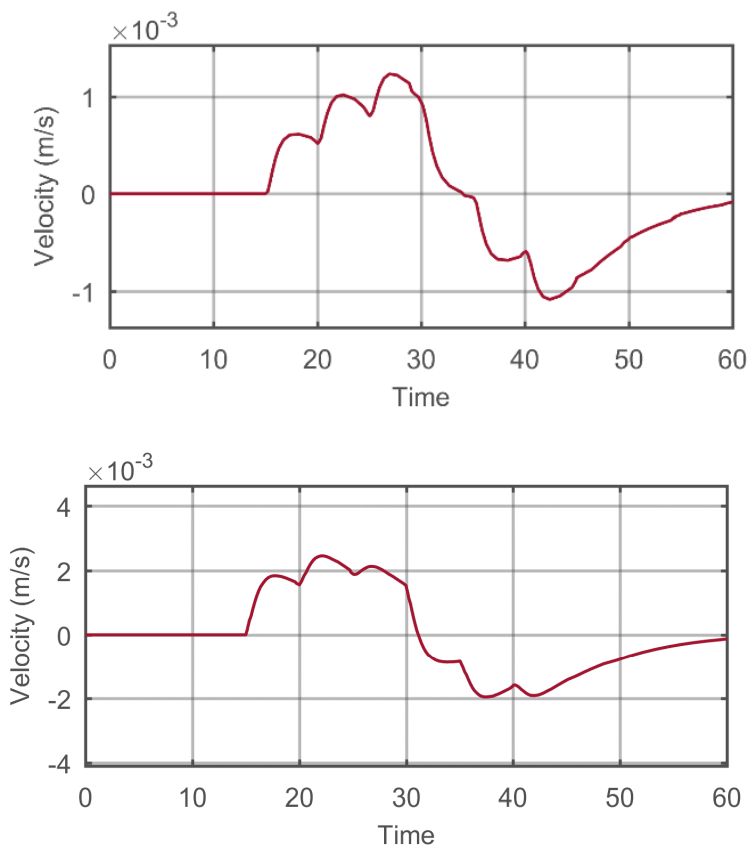

(b)

(b)

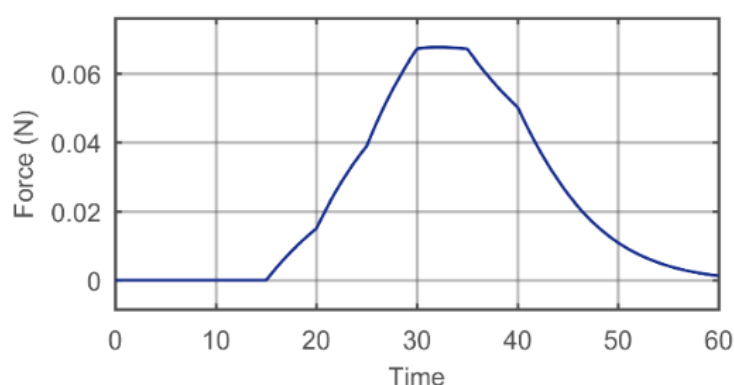

(c)

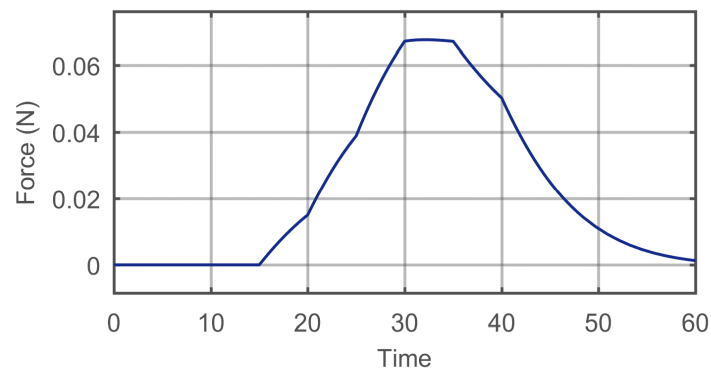

(c)
Fig. 13. Modules combined arrangement results at $\tau_{1,} \tau_{7}=5 \mathrm{sec}$, (a) Displacement (b) Velocity (c) Force.
Fig. 14. Modules separate arrangement results at $\tau_{1}, \tau_{2}=5$ sec. (a) Displacement (b) Velocity (c) Force. 
DEVELOPMENT OF MULTI-MODULE ARTIFICIAL MUSCLE USING HYBRID ACTUATOR
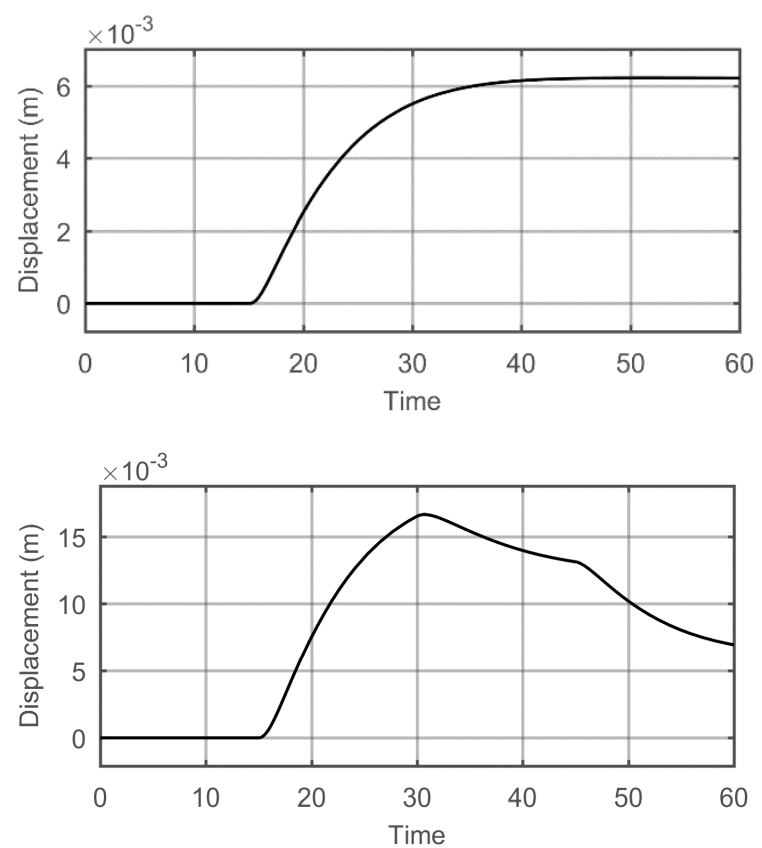

(a)

(a)
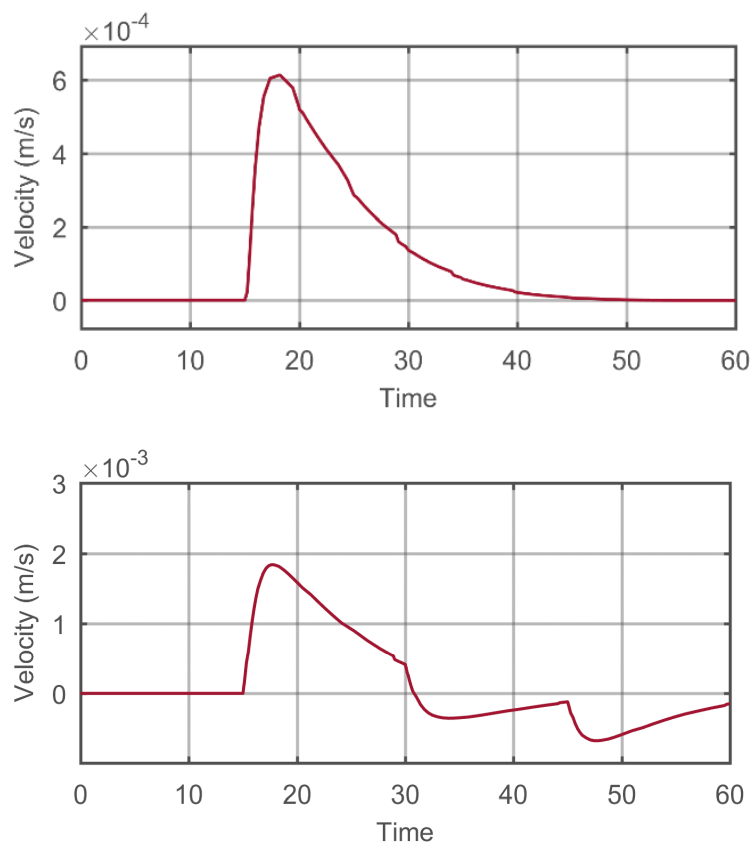

(b)

(b) 

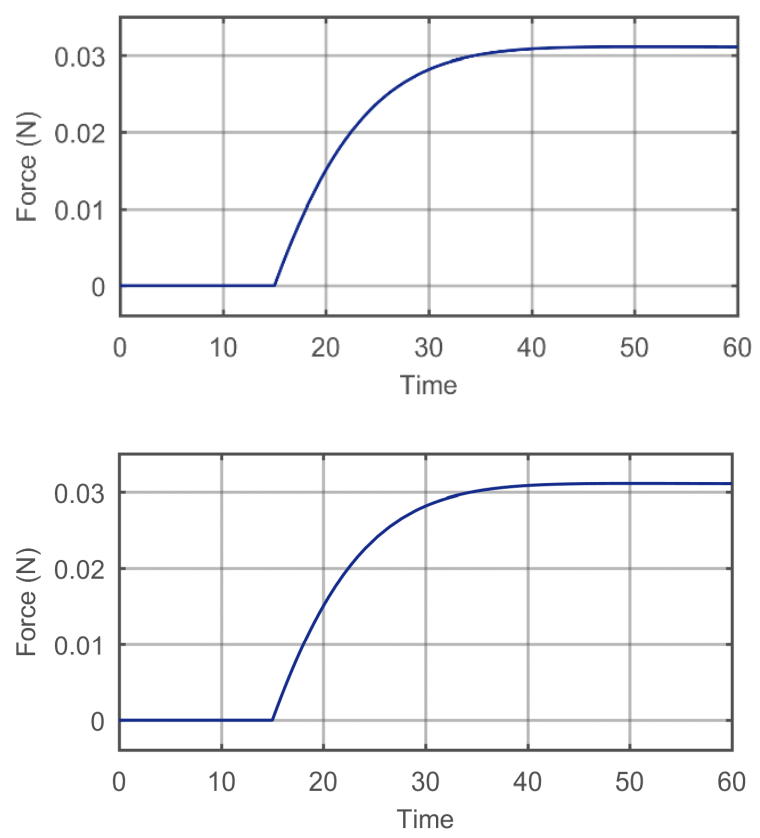

(c)

Fig. 15. Modules combined arrangement results at $\tau_{1}, \tau_{2}-15 \tau_{i} \tau_{-}$(a) Displacement (b) Velocity (c) Force.
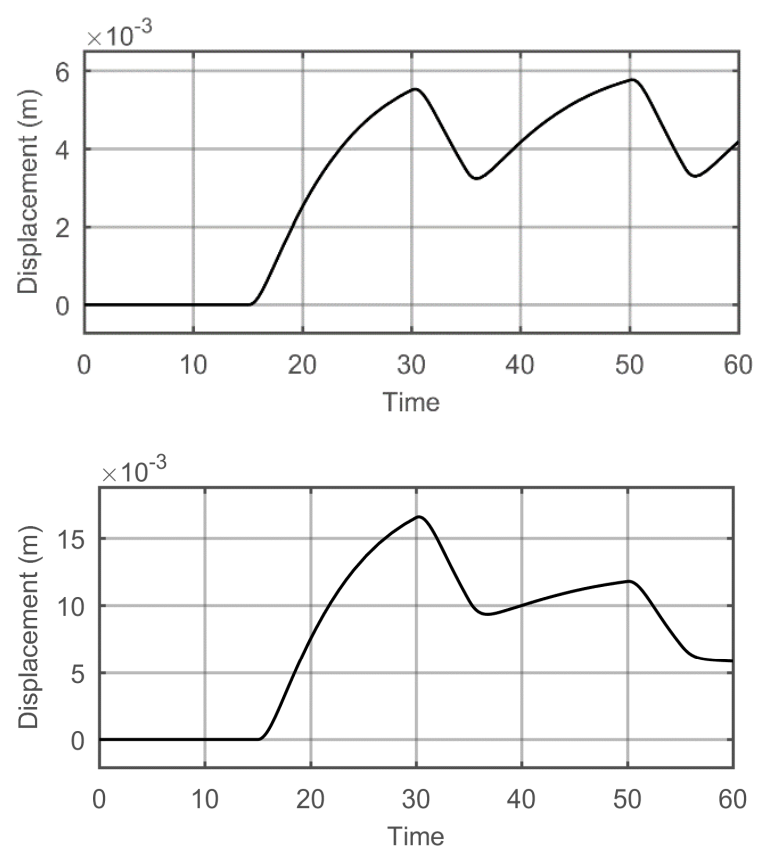

(a) (c)

Fig. 16. Modules separate arrangement results at $\tau_{1}, \tau_{2}-1 r_{1} s_{r}$..(a) Displacement (b) Velocity (c) Force. (a) 

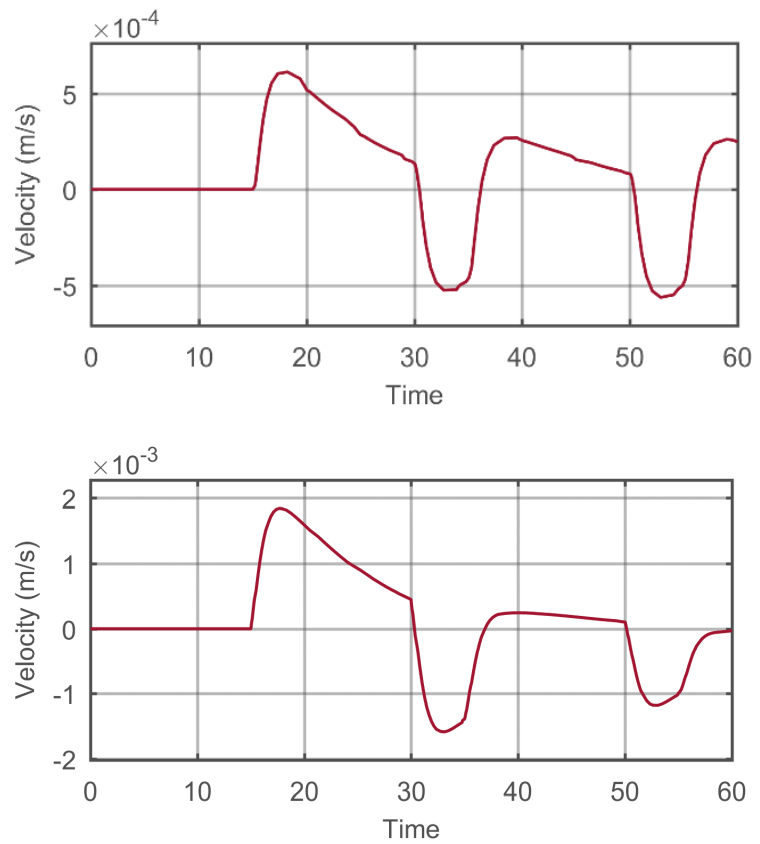

(b)

(b)
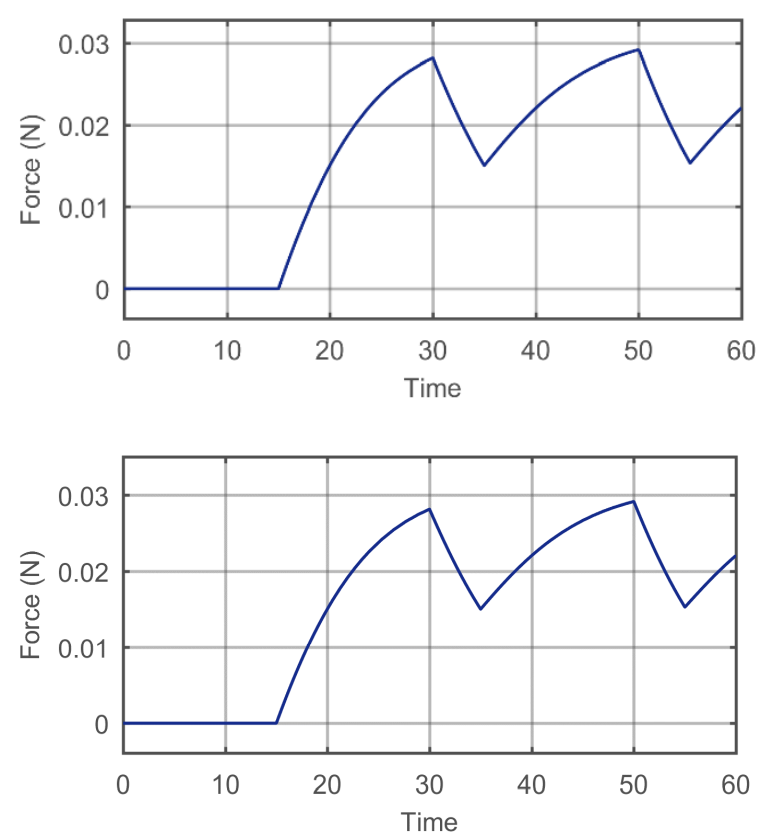

(c)

(c)

Fig. 17. Modules combined arrangement results at $\tau_{1,} \tau_{7}=20$ sec. (a) Displacement (b) Velocity (c) Force.

Fig. 18. Modules separate arrangement results at $\tau_{1}, \tau_{2}=15$ sec. (a) Displacement (b) Velocity (c) Force.

According to the previous graphs, the proposed combinations shows a significant improvement in the force, displacement, and velocity values compared to that of the inspiring design in [28]. The resultant force using combined arrangement with no time lag $\left(\tau_{1}, \tau_{2}=0\right)$ gives $0.08 \mathrm{~N}$ relative to 0.06 in the previous design. Displacement reaches $6 \mathrm{~mm}$ relative to 
$2.5 \mathrm{~mm}$ in the previous design. Finally, the velocity didn't show significant change in the proposed design.

\section{CONCLUSION AND FUTURE WORK}

Inspired by the four-step cycle of the natural sarcomere, this work presents an autonomous, hybrid material, artificial multi-module sarcomere. The beating hydrogel heads attached to modules are powered by the BZ chemical reaction, which in turn enables the IPMC strips to bend giving forward displacement to the attached sliders. The modules were grouped into two proposed arrangements; the combined arrangement in which all modules are working at the same time, and the separate module in which modules are working in sequence.

A mathematical model was extracted and simulated by MATLAB Simulink and both arrangement performance criteria were analyzed. The actuator arrangements were able to enhance the actuator performance, giving larger force output for the combined arrangement and larger displacement for separate arrangement. Also increasing time delay between modules enables the actuator to maintain the force for a pre-determined time.

The proposed configuration results are promising to cover many varieties of desired outputs since they can be recruited in larger combinations to mimic the natural muscle behavior.

\section{REFRENCES}

1. Nomura, M. T. Sh-hei, Volkenburgh E.V., Mizunami M.,2016, Bioinspired Actuators and Sensors. Cambridge University Press.

2. Abdelwahab, S. A.Tolbah F.A., 2015, "Design and Development of a Motion Control System Based on Human Like Actuator and IP," International Workshop on Research and Education in Mechatronics (REM). pp9-11.

3. Peng, C. Peng, Yin Y. H., Hong H. B., Zhang J. J, and Chen X.,2017, "Bio-inspired design methodology of sensor-actuator-structure integrated system for artificial muscle using SMA," Procedia CIRP, vol. 65, pp. 299-303.

4. Hilber, W, 2016, "Stimulus-active polymer actuators for next-generation microfluidic devices," Applied Physics A: Materials Science and Processing, vol. 122, no. 8, pp. 139.

5. Bar-cohen, Y, 2002, "Electroactive Polymers as Artifcial Muscles: A Review Introduction," vol. 39, no. 6.

6. Tolbah, F.A., Abdelhameed M. M., Awad M. I., and Abdelwahab, S. A., 2014, "Modeling and simulation of a new bioinspired muscle actuator," 15th International Workshop on Research and Education in Mechatronics, REM.

7. Seeley, C., Rod; Russo, Andy, Regan, Jennifer; Putto V.,2014 Anatomy \& Physiology. Wiley.

8. https://www.pearson.com/content/dam/one-dot-com/one-dot-com/us/en/highered/en/products-services/course-products/marieb-10e-info/pdf/marieb-0321927028chapter9.pdf.

9. Jovanovic, K., Vranic J., and Miljkovic N. ,2015, "Hill's and Huxley's muscle models - tools for simulations in biomechanics," Serbian Journal of Electrical Engineering, vol. 12 , no. 1 , pp. 53-67.

10. Lymn, R. W. and Taylor E. W., 1971, "Mechanism of Adenosine Triphosphate Hydrolysis by Actomyosin," Biochemistry, vol. 10, no. 25, pp. 4617-4624.

11. Chen, J, Zhang X., Lin S., Wang H., and Gu L., 2015, "Multiscale modeling of skeletal muscle active contraction in relation to mechanochemical coupling of molecular motors," Micromachines, vol. 6, no. 7, pp. 902-914.

12. Chen, J., Zhang X., and Wang H., 2015, "Research on Biomechanical Model of Muscle Fiber Based on Four-State Operating Mechanism of Molecular Motors," pp. 529-532.

13. Kull, F. J. and Endow S. A., 2013, "Force generation by kinesin and myosin cytoskeletal motor proteins," Journal of Cell Science, vol. 126, no. 1, pp. 9-19.

14. Hudson, T. A., Bragg J. A., Brown E. A., Lin D. C., and Deweerth S. P., 1999, "Development of a sarcomere model using hardware," The Erst Joint BMES/EMBS Conference Serving Humanity vol. 57, no. 2, p. 7803. 
15. Hudson, T. A., Bragg J. A., Brown E. A., Lin D. C., and Deweerth S. P., 2000, "an analog VLSI model of muscle sarcomeres," 2000 IEEE International Symposium on Circuits and Systems. Emerging Technologies for the 21st Century. Proceedings (IEEE Cat No.00CH36353), vol. 1, no. x, pp. 431-434.

16. Hudson, T. A., Bragg J. A., Brown E. A., Lin D. C., and Deweerth S. P., 2001, "an integrated circuit implementation of the Huxley sarcomere model," IEEE Transactions on Biomedical Engineering, vol. 48, no. 12, pp. 1471-1479.

17. Ebrahimi, N., Nugroho S., Taha A. F., Gatsis N., Gao W, and Jafari A., 2018, "Dynamic Actuator Selection and Robust State-Feedback Control of Networked Soft Actuators," International Conference on Robotics and Automation (ICRA), vol. 1.

18. Schultz, J., Mathijssen G., Vanderborght B., and Bicchi A., 2014, "Toward motorunit-recruitment actuators for soft robotics," Proceedings of the IEEE RAS and EMBS International Conference on Biomedical Robotics and Biomechatronics, pp. 887-892.

19. Schultz, J., Mathijssen G., Vanderborght B., and Bicchi A., 2015, "A selective recruitment strategy for exploiting muscle-like actuator impedance properties," IEEE International Conference on Intelligent Robots and Systems, vol. 2015-Decem, pp. 2231-2237.

20. Jing, L., Xiansheng Q., Xuefeng Z., and Zhanxi W., 2011, "Multi-object optimal design of electromagnetic artificial muscle structure," International Conference on Transportation, Mechanical, and Electrical Engineering (TMEE), pp. 1152-1156.

21. Jing, L., Xiansheng Q., Xuefeng Z., and Zhanxi W., 2017, "Linear Electromagnetic Array Artificial Muscle Design and Simulation for a Quadruped Robot," Journal of Mechanics in Medicine and Biology, vol. 17, no. 7.

22. Wang, T., Huang J., Yang Y., Zhang E., Sun W., and Tong Z., 2015, "Bioinspired Smart Actuator Based on Graphene Oxide-Polymer Hybrid Hydrogels," ACS Applied Materials and Interfaces, vol. 7, no. 42, pp. 23423-23430, 2015.

23. Hamdi, H M, Tolbah F. A., Abdelwahhab S A, Awad M I, 2020, "Design of bioinspired muscle sarcomere structure using a hybrid Hydrogel-IPMC actuator," in International Conference on Applied Mechanics and Mechanical Engineering AMME19.

24. Calvert, P., 2008, "Gel Sensors and Actuators," MRS Bulletin, vol. 33, no. 03, pp. 207-212.

25. Ballhause, D. and Wallmersperger T., 2008, "Coupled chemo-electro-mechanical finite element simulation of hydrogels : I . Chemical stimulation," vol. 045011.

26. Yoshida, R., Kokufuta E., T. Yamaguchi, and Yamaguchi T., 1999, "Beating polymer gels coupled with a nonlinear chemical reaction Beating polymer gels coupled with a nonlinear chemical reaction," Chaos: An Interdisciplinary Journal of Nonlinear Science, vol. 9, pp 260-266.

27. Yoshida, R. and Ueki T., 2014, "Evolution of self-oscillating polymer gels as autonomous polymer systems," NPG Asia Materials, vol. 6, March, pp. 1-14.

28. Maeda, S., Hashimoto S., and Yoshida R., 2004, "Design of chemo-mechanical actuator using self-oscillating gel," IEEE International Conference on Robotics and Biomimetics vol. c, pp. 474-479.

29. Yoshida, R., 2010, "Design of Self-Oscillating Gels and Application to Biomimetic Actuators," Sensors, vol. 10, pp. 1810-1822.

30. Vokoun, D., He Q., Heller L. Yu M., and Dai Z., 2015, "Modeling of IPMC Cantilever's Displacements and Blocking Forces," Journal of Bionic Engineering, vol. 12 , no. 1, pp. 142-151.

31. Kazem, B. and Khawwaf J., 2016, "Estimation Bending Deflection in an Ionic Polymer Metal Composite (IPMC)" Jordan Journal of Mechanical \& Industrial Engineering, vol. 10, no. 2, pp. 123-131.

32. Kim, K., 2016, "Electromechanically Active Polymers," Electromechanically Active Polymers, Wiley.

33. Ruiz, S. A., 2013, "3D Modeling and Design Optimization of Rod Shaped Ionic Polymer Metal Composite Actuator," UNLV Theses, Dissertations, Professional Papers, and Capstones. 2829. 
34. Yashin, V. V. and Balazs A.C., 2014, "Theoretical and computational modeling of self-oscillating polymer gels Theoretical and computational modeling of selfoscillating polymer gels," The Journal of chemical Physics, no.126, pp 1-18.

35. Yashin, V. V, Levitan S. P., Balazs A. C., S. P. Levitan, and A., 2015, "Modeling the entrainment of self-oscillating gels to periodic mechanical deformation," Choas, vol. 064302, no 25, pp 1-10.

36. Moeinkhah, H., Rezaeepazhand J., and Akbarzadeh A., 2013, "Analytical dynamic modeling of a cantilever IPMC actuator based on a distributed electrical circuit," Smart Materials and Structures, vol. 22, no. 5.

37. Wang, Z., He B., Liu X., and Wang Q., 2017, "Development and modeling of a new ionogel based actuator," Journal of Intelligent Material Systems and Structures, vol. 28, no. 15, pp. 2036-2050.

38. Dougal R. A., Gao L., and Liu S., 2004, "Ultracapacitor model with automatic order selection and capacity scaling for dynamic system simulation," Journal of Power Sources, vol. 126, no. 1-2, pp. 250-257.

39. Shi, L., Guo S., and Asaka K., 2012, "Modeling and experiments of IPMC actuators for the position precision of underwater legged microrobots," IEEE International Conference on Automation and Logistics, ICAL, no. August, pp. 415-420.

40. Gong, Y., Fan J., Yin, Tang C., and pong C., 2011, "Numerical simulation of dynamic electro-mechanical response of ionic polymer-metal composites," Journal of Bionic Engineering, vol. 8, no. 3, pp. 263-272. 